\title{
Was the Day Hospital worthwhile?
}

\section{Meira DA (1)}

(1) Professor Emeritus of the Botucatu Medical School, São Paulo State University, UNESP, Botucatu, São Paulo State, Brazil and Technical Director of the Domingos Alves Meira Day Hospital, Medical and Hospital Development Foundation, FAMESP, Botucatu, São Paulo State, Brazil.

Dear Editor,

In August, 1994, during the X Conference on AIDS, held in Yokohama, Japan, Stefano Vella presented the action of a protease inhibitor on HIV in a multicenter study conducted by him. On that occasion, he showed micrographs of the virus that had been influenced by this drug, revealing an empty capsid, that is, without the nucleic-acid helices. In these conditions, they changed into "virions" that are incapable of infecting cells, thus interrupting the vital cycle of HIV. In the same presentation, the author referred to tuberculosis treatment that only became effective with the beginning of the triple scheme, as it, in a certain way, reduced the phenomenon of bacterial resistance to tuberculostatics. The associated use of two reverse transcriptase inhibitors with the protease inhibitor was then postulated. Such therapeutic scheme became known as the "triple scheme" or "cocktail".

By means of the National STD/AIDS Program, the Ministry of Health then offered this therapeutic scheme to all HIV-infected individuals residing in Brazil, and it has been adopted since 1996.

From the following year, important alterations were observed in the development of the disease, including increased survival, improved control of opportunistic infections and better quality of life. As a consequence of these changes, patients' needs for hospitalization have been significantly reduced. On the other hand, the demand for clinics and outpatient units to provide care for these persons considerably 
augmented. This scenario led the Ministry of Health to suggest the Day Hospital as an institution model to meet patient demands.

On that occasion, the suggestion seemed to be promising, since it would enable to direct the architectural conception as well as the construction engineering of the Day Hospital to the final aspect of one-storey houses, surrounded by a garden with flowers and fruit trees, thus providing patients with comfort, privacy, with a separate waiting area, but without the physical barrier of the care provision and procedure wings. The Day Hospital has eight common consulting rooms and areas for specific physiotherapeutic care, occupational therapy, pre- and post-nursing consultation, psychological care, psychiatric care, social assistance, proctologic care, gynecological care, nutritional correction (dyslipidemia) and corrective plastic surgery for lipodystrophy. There is also an area for group meetings in addition to a dental office, a pharmacy, ultrasonography services and three apartments for one-day inpatients. This configuration allows for a quiet and calm environment, excellent to receive patients as well as adequate for professionals to work in.

The patient care includes diagnostic confirmation, general health assessment and treatment. The multiprofessional team, however, is concerned not only about the physical treatment, but also about mental, behavioral and psychopathological aspects, which correspond to a large number of problems presented by the patients, who are affected by prejudice, discrimination and stigma. These aggravations frequently lead them to affective problems, anxiety disorders and, not rarely, to suicidal ideas. In the Day Hospital, the multiprofessional performance presents good outcomes in patients' resocialization and even in their inclusion into the labor market. With regard to the quantitative aspect, a steady increase in the number of patients seeking the Day Hospital has been observed. Such augment is partly due to the expansion to Botucatu region. Hence, since this entity began to operate on September $9^{\text {th }}, 2004$, approximately 64,000 consultations have been recorded. Such consultations include patients infected with HIV-I, chronic hepatitis by B and C viruses, victims of accidents caused by biological material or sexual harassment as well as individuals infected with the HTLV-I and II viruses.

The set of factors that characterize the Day Hospital allows patients to feel more confident and secure, which makes it an extension of their homes. This has led to greater adherence to treatment, from 70.6\%, when care was provided at the University Hospital Outpatient Unit, to $96.6 \%$ since the Day Hospital was instituted. 
Similarly, and in the same periods of observation, absenteeism, which was previously frequent, no longer exists in the Day Hospital. This may be even due to the possibility of consultations at shorter intervals.

The results achieved in the last five years of care provision at the University Hospital were also compared to those obtained in the first five years at the Day Hospital, and the following figures were respectively found: 931 and 867 patients infected with HIVI were registered; 3,803 and 7,307 consultations were performed; 363 and 70 deaths occurred. These results are highly significant and foster the work developed at the Day Hospital, a place where care and treatment are provided without disregarding the importance of humanization.

It is for all these reasons that the following conclusion can be enthusiastically reached: yes, the creation of the Day Hospital was worthwhile!

\section{CORRESPONDENCE TO:}

DOMINGOS ALVES MEIRA, Hospital Dia "Domingos Alves Meira", Alameda dos Cedrinhos, 54, Distrito de Rubião Júnior, Botucatu, SP, 18618-000, Brasil. Phone: +55 143811 6537. Email: d.meira@uol.com.br. 